\title{
LA-UR-12-22337
}

Approved for public release; distribution is unlimited.

Title:

Author(s):

Intended for:

\section{SpAMM - The Power of $\mathrm{N}$}

Bock, Nicolas

Challacombe, William M.

Web

\section{- Losalamos

\author{
EST. 1943
}

Disclaimer:

Los Alamos National Laboratory, an affirmative action/equal opportunity employer,is operated by the Los Alamos National

Security, LLC for the National NuclearSecurity Administration of the U.S. Department of Energy under contract DE-AC52-06NA25396.

By approving this article, the publisher recognizes that the U.S. Government retains nonexclusive, royalty-free license to

publish or reproduce the published form of this contribution, or to allow others to do so, for U.S. Government purposes.

Los Alamos National Laboratory requests that the publisher identify this article as work performed under the auspices of the

U.S. Departmentof Energy. Los Alamos National Laboratory strongly supports academic freedom and a researcher's right to publish; as an institution, however, the Laboratory does not endorse the viewpoint of a publication or guarantee its technical correctness. 


\section{SpAMM - The power of $N$}

Database

SpAMM

Gaming

Operations

\section{Generalized N-Body Solvers}

Computer Graphics

\section{Culling}

Science

Sparse/Irregular FMM/HOT

- Linear scaling complexity, $\mathrm{O}(\mathrm{N})$

- With scalable parallelism, increasing core count yields proportional capability gains

Machine Learning 


\section{SpAMM - The power of $N$}

- Sparse Approximate Matrix Multiply (SpAMM) for matrices with decay

- Linear scaling electronic structure (FreeON)

- N-Body learning

- General alternative to incomplete matrix algebra ("sparsification")

- On the fly dropping of product contributions can lead to better accuracy than GEMM, and $\mathrm{O}(\mathrm{N})$ execution time for matrices with decay. 


\section{SpAMM - The power of $N$}

\section{SP2/SpAMM}

\section{FreeON}

$\mathrm{HiCu}$

http://www.freeon.org

ONX

QCTC 


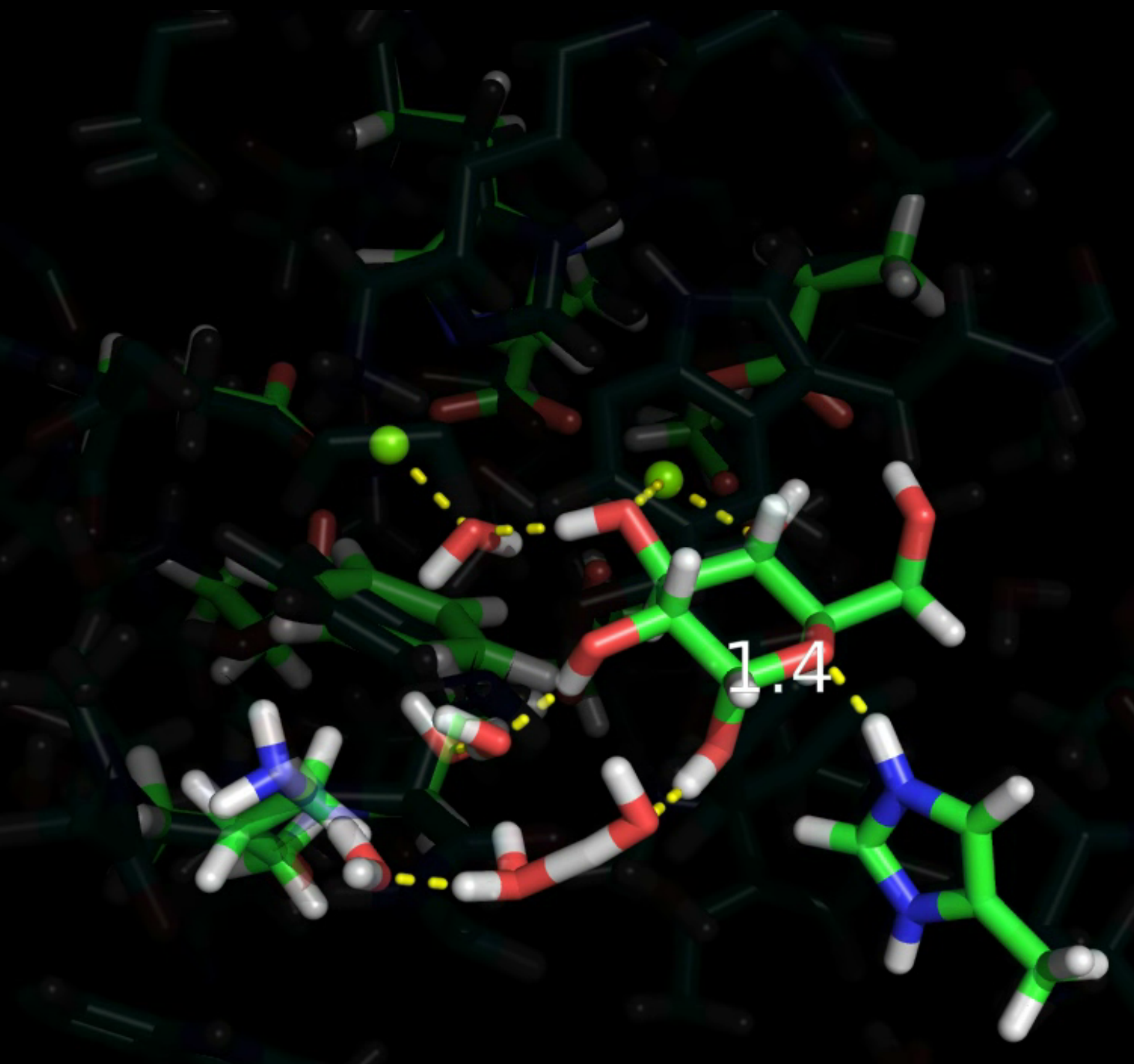




\section{SpAMM - The power of $N$}
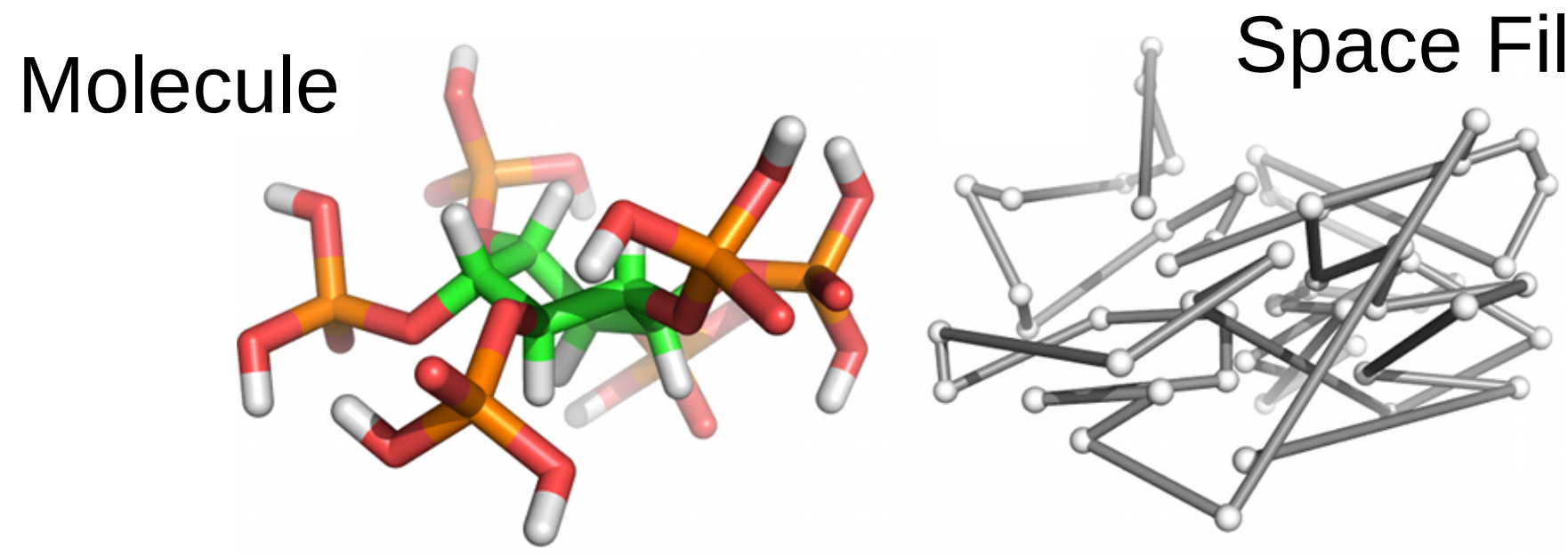

Matrix/Quadtree

\begin{tabular}{|c|l|l|}
\hline 0000 & 0001 & \\
0010 & 0011 & \\
& 01 \\
& & \\
10 & 11 \\
\end{tabular}

Convolution/Octree
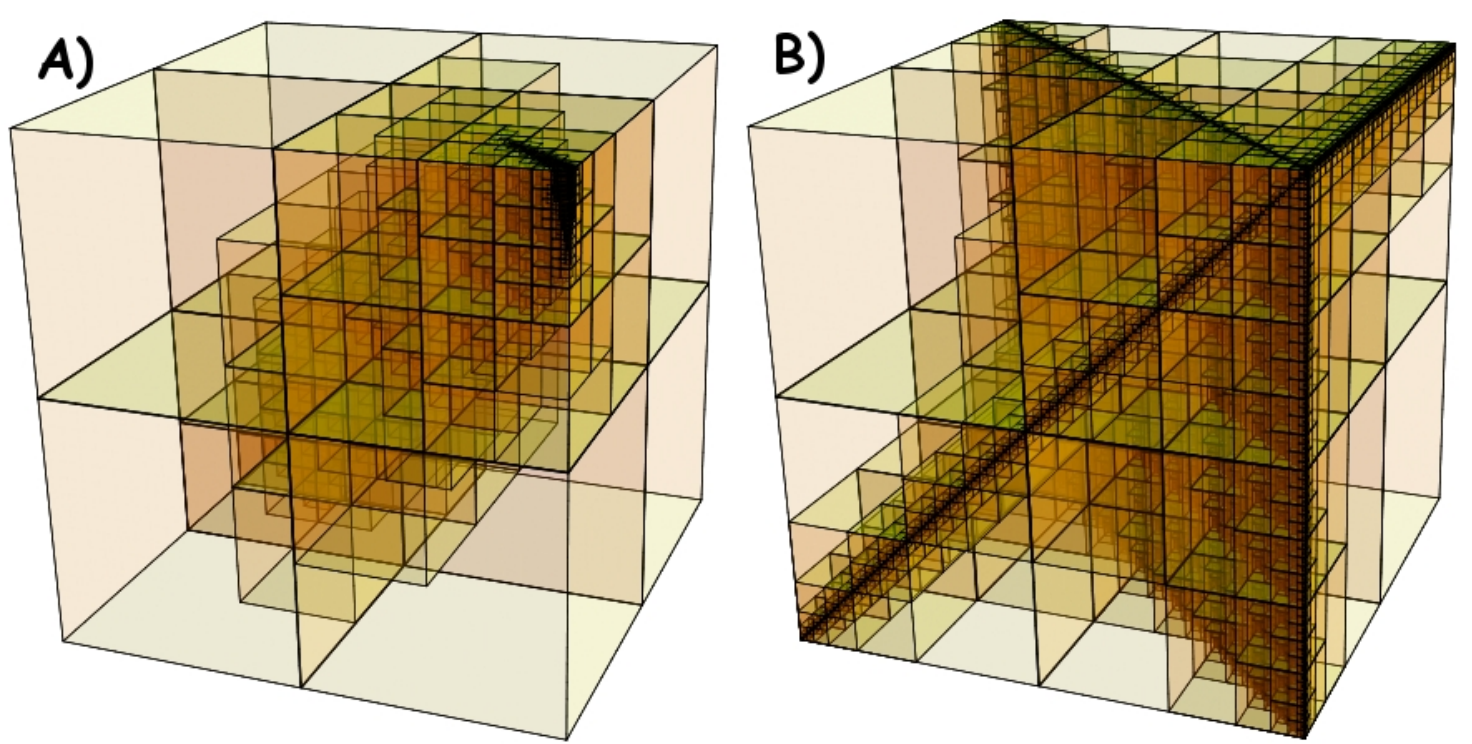

A) Exponential decay, B) Algebraic decay 


\section{SpAMM - The power of $N$}

- Linked list on top tiers $\rightarrow$ recursive execution

- Linear quadtree on bottom tiers

- Hashtables/Linear index

- Handcoded assembly kernel for multiply

- Task parallelism with OpenMP 3.0 on top

- High performance serial execution on bottom

- Dropping is applied all the way down to 4x4

- Non-contiguous, dynamic allocation 


\section{SpAMM - The power of $N$}

- Effective serial performance of SpAMM relative to SGEMM - For $2 \times 10^{-8}$ the max-norm error matches SGEMM

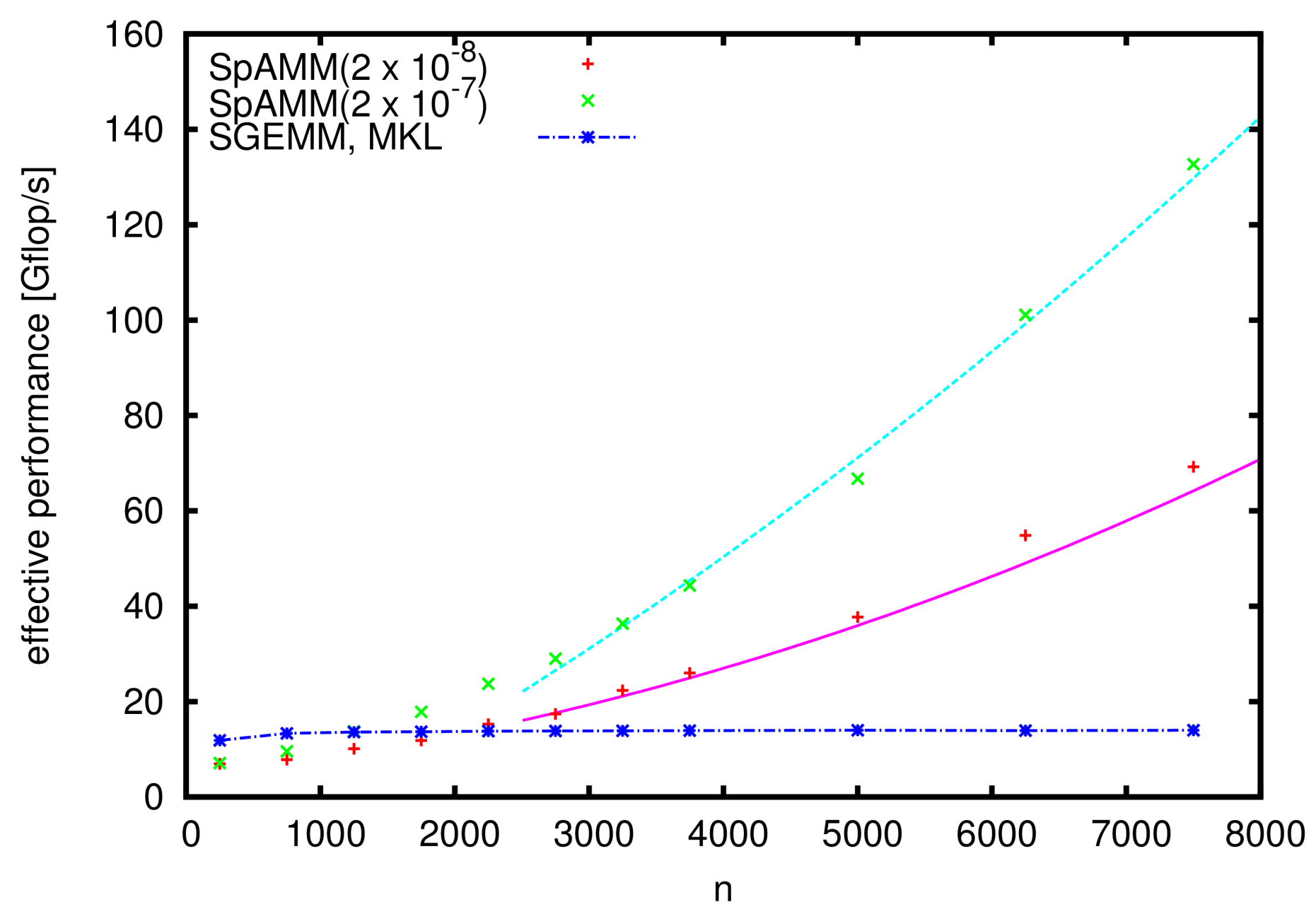




\section{SpAMM - The power of $N$}

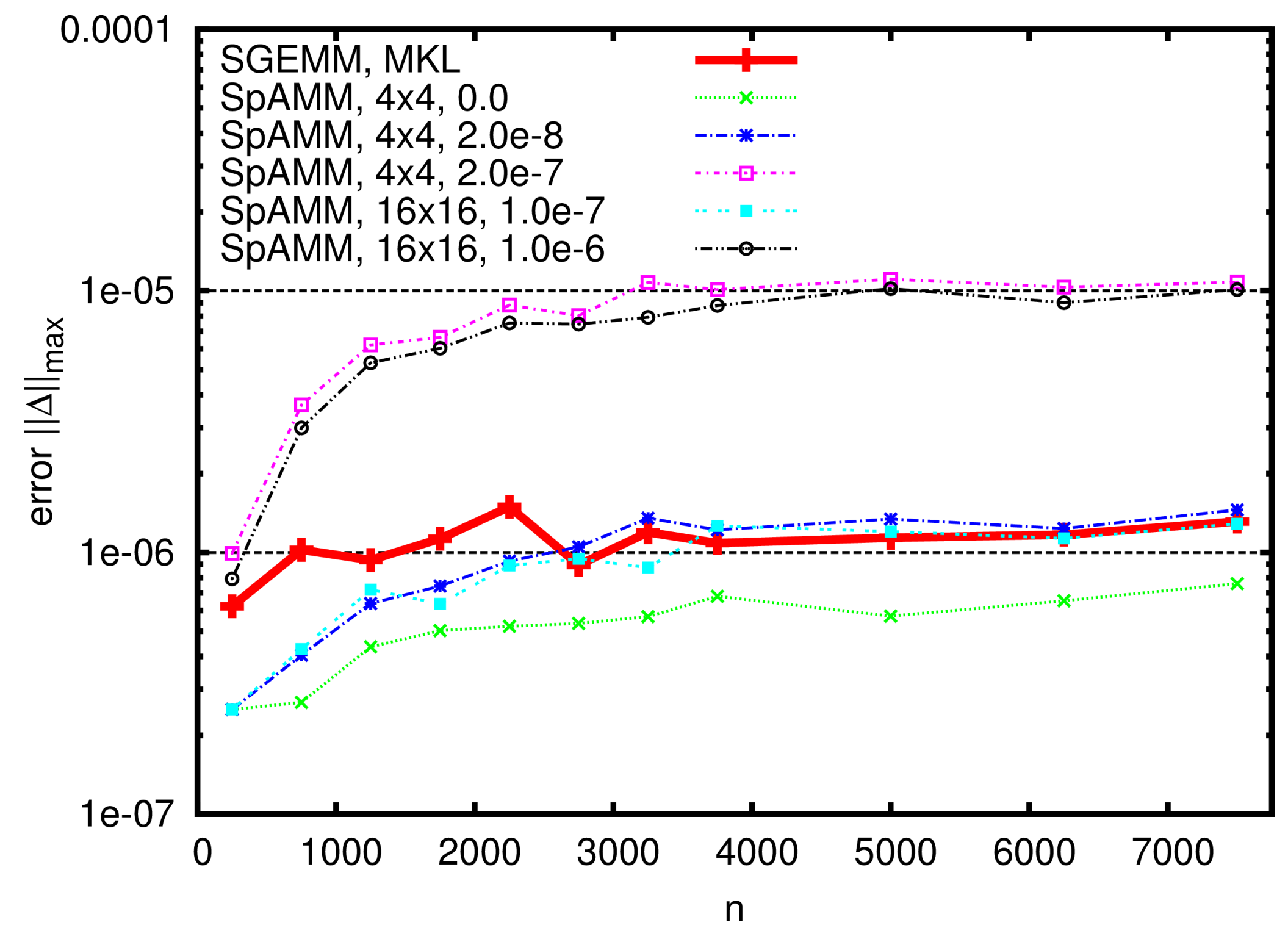




\section{SpAMM - The power of $N$}

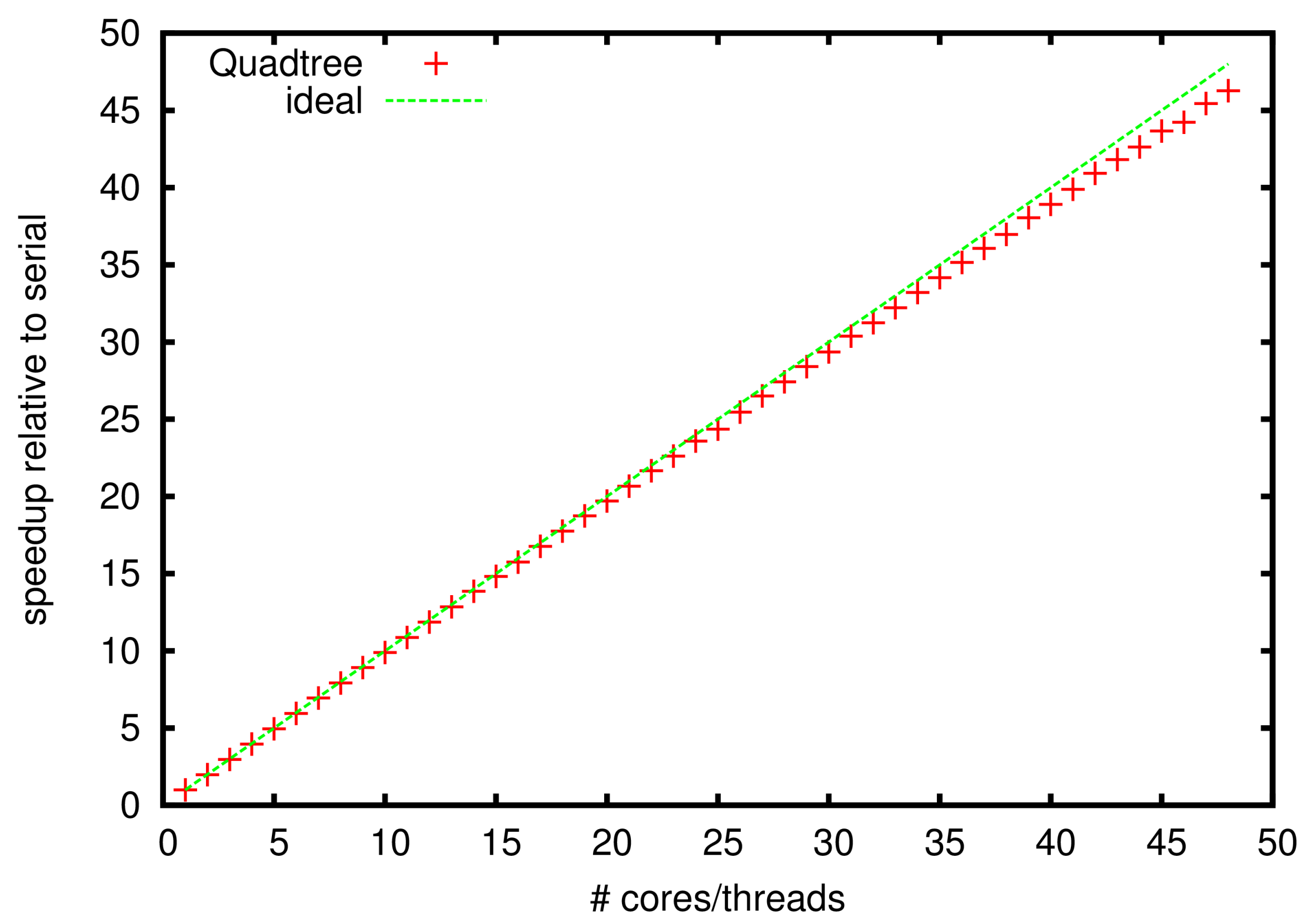




\section{SpAMM - The power of $N$}

Goals for MIC

- Achieve close to ideal speedup on MIC with task parallel paradigm

- Improve data prefetch on fine granularity multiply kernel

Nicolas Bock

Matt Challacombe 